\title{
Aspectos Neuropsicológico e Neurológico de Crianças Nascidas Prematuras e com Peso Inferior a 1.500 gramas
}

\author{
Neurological and Neuropsychological Aspects of Children Born Preterm \\ and with Weight Lower than 1,500 grams
}

\author{
Alexandre Ferreira Campos ${ }^{*}, a$, Leandro Fernandes Malloy-Diniz ${ }^{a}$, \\ Jane de Almeida Nascimento ${ }^{b} \&$ Regina Helena Caldas de Amorim $^{a}$ \\ ${ }^{a}$ Universidade Federal de Minas Gerais, Belo Horizonte, Brasil \\ $\&{ }^{b}$ Universidade FUMEC, Belo Horizonte, Brasil
}

\begin{abstract}
Resumo
Crianças nascidas prematuras, especialmente aquelas com peso $<1.500 \mathrm{~g}$, apresentam frequentemtente complicações neonatais e riscos para problemas de desenvolvimento. Nesse estudo, 20 crianças com inteligência e exame neurológico normal, aos sete anos, nascidas $<33$ semanas de gestação e peso $<1.500 \mathrm{~g}$, foram comparadas com 20 crianças nascidas a termo. Avaliou-se a inteligência pela WISC-III e o comportamento pelo Questionário de Conners. O Exame Neurológico Evolutivo-Lefèvre foi aplicado nas prematuras. As crianças prematuras apresentaram nível intelectual inferior ao das nascidas a termo. No Conners não houve diferença entre os grupos. Apenas $20 \%$ das prematuras completaram todas as provas do Exame Evolutivo previstas para a idade. Os dados alertam para a necessidade de avaliações específicas, na idade escolar, em crianças nascidas prematuras, mesmo que apresentem exame neurológico normal. Palavras-chave: Prematuridade; Baixo Peso ao Nascer; Exame Neurológico; Inteligência; Comportamento.
\end{abstract}

\begin{abstract}
Preterm children - especially those with birth weight $<1,500 \mathrm{~g}$ - often present neonatal complications and higher risk of suffering from developmental disorders. Twenty children aged seven, with average intelligence and normal neurological exams, born with gestational age $<33$ weeks and weight $<1,500 \mathrm{~g}$, were enrolled in a study and compared with 20 children born at full term. Intelligence was measured by WISCIII and behavior by the Conners Questionnaire. Lefèvre Evolutive Neurological Examination was applied in the preterm children. Children born preterm had lower IQ than those born at full term. In the Conners, there was no difference between groups. Only $20 \%$ of preterm children performed all tests from the Evolutive Exam for their age. This study highlights the importance of specific cognitive evaluations in preterm children during school age, even if they have normal neurological exams.

Keywords: Preterm; Low Birth Weight; Neurologic Examination; Intelligence; Behavior.
\end{abstract}

A prematuridade (recém-nascido de uma gestação $<37$ semanas) e o baixo peso $(>1.000 \mathrm{~g}$ e $<2.500 \mathrm{~g})$, em especial o muito baixo peso $(<1.000 \mathrm{~g})$, conforme definição da Organização Mundial da Saúde (OMS, 1998), quando associados, potencializam problemas clínicos e frequentemente afetam de modo negativo o desenvolvimento neuropsicomotor e cognitivo. Crianças nascidas prematuras, principalmente aquelas com peso abaixo de $1.500 \mathrm{~g}$, apresentam, com maior frequência, complicações neonatais, pior desempenho cognitivo e escolar e distúrbios de crescimento (Cooke \& Foulder-Hughes, 2003; Esbjorn, Hansen, Greisen, \& Mortensen, 2006; Fily et al., 2003; Luu et al., 2009; McGrath \& Sullivan, 2002;

\footnotetext{
" Endereço para correspondência: Avenida do Contorno, 3979, sala 404, São Lucas, Belo Horizonte, MG, Brasil, CEP 30110-090. Tel.: (031) 3223 8053. E-mails: alexfercam@ig.com.br, malloy.diniz@gmail.com, anean@bol.com.br e hcamorim@terra.com.br
}

Saigal et al., 2003). Segundo Cooke (2005), em 10\% a $15 \%$ das crianças nascidas antes de 32 semanas de idade gestacional (IG) observa-se algum tipo de deficiência; cerca de $40 \%$, quando inseridas em educação inclusiva, têm problemas motores, de comportamento ou de aprendizagem. Esse autor mencionou ainda que, mesmo na ausência de deficiência intelectual ou déficits cognitivos globais, observam-se alterações cognitivas ou comportamentais específicas.

Mcgrath et al. (2005) e Shum, Neulinger, O'Callaghan e Mohay (2008) afirmaram que há maior ocorrência de sintomas de desatenção e hiperatividade em crianças nascidas prematuras e com baixo peso, em comparação às crianças nascidas a termo e com peso adequado à idade gestacional (AIG). A prevalência de Transtorno do Déficit de Atenção/Hiperatividade (TDAH) na população escolar varia entre $3 \%$ a $7 \%$, sendo até quatro vezes maior em crianças prematuras (Aylward, 2005; Böhm, Smedler, \& Forssberg, 2004). Além da maior frequência 
de transtornos comportamentais, déficits relacionados à memória episódica e às funções executivas em crianças prematuras têm sido igualmente evidenciados (Böhm et al., 2004; Briscoe, Gathercole, \& Marlow, 2001). Mesmo em situações nas quais o exame neurológico tradicional é normal, disfunções neurológicas muitas vezes sutis puderam ser constatadas. Por exemplo, Breslau, Chilcoat, Johnson, Andreski e Lucia (2000) avaliaram, aos seis anos de idade, 823 crianças nascidas com baixo peso nos Estados Unidos, utilizando a Neurologic Evaluation Schedule (Hertzig, 1987), e localizaram sinais neurológicos leves. Aos seis anos de idade, as crianças também foram submetidas à avaliação neuropsicológica, que incluiu a WISC-R (Wechsler, 1974), uma avaliação global das competências da criança (Rourke, 1985; Rourke, Fisk, \& Strang, 1986) e medidas do comportamento, que tiveram como base o relato dos pais (Child Behavior Checklist) e dos professores (Teacher Report Form; Achenbach, 1991a, 1991b). A avaliação neuropsicológica foi repetida aos 11 anos, com a inclusão de informações sobre o desempenho escolar e medidas do rendimento acadêmico - Woodcock-Johnson Psycho-Educational Battery-Revised (Woodcock \& Johnson, 1989). As crianças que apresentaram mais sinais neurológicos leves aos seis anos, tiveram pior desempenho cognitivo, mais problemas comportamentais e menor aproveitamento acadêmico, tanto aos seis, quanto aos 11 anos.

Isaacs, Edmonds, Lucas e Gadian (2001) avaliaram a inteligência, o raciocínio matemático e a leitura, por meio dos testes WISC-III (Wechsler, 1992), Wechsler Objective Numeric Dimensional Test (Wechsler, 1996) e Wechsler Objective Reading Dimensions (Wechsler, 1993), de 80 adolescentes nascidos com peso de até $1.500 \mathrm{~g}$ e $\mathrm{IG}<30$ semanas, sem alterações neurológicas clínicas. Os autores encontraram dificuldades específicas em cálculos aritméticos e concluíram que esses déficits devem estar associados às disfunções no lobo parietal esquerdo, detectadas na Ressonância Nuclear Magnética.

Em estudo prospectivo realizado na Inglaterra por Allin et al. (2006) com 153 jovens de 18 anos, nascidos antes de 33 semanas de gestação, foi constatado que eles apresentavam alguma alteração, em uma ou mais das escalas de Griffiths (Griffiths, Sigmundsson, Takei, Rowe, \& Murray, 1998), que é uma versão modificada da Escala de Avaliação Neurológica de Buchanan e Heinrichs (Buchanan \& Heinrichs, 1989), efetuadas aos 12 meses, aos quatro e aos oito anos. Os problemas detectados por essas escalas, na infância, foram classificados como primários e sinais integrativos. Os primários correspondem àqueles identificados habitualmente pelo exame neurológico tradicional, e os sinais integrativos, os que requerem integração no sistema motor, ou entre os sistemas motor e sensorial. Aos 18 anos, esse grupo foi avaliado por meio da Wechsler Adult Intelligence Scale - Revised (Wechsler, 1981). Os distúrbios neurológicos identificados na infância, ainda que discretos, foram relacionados a pior desempenho no teste neuropsicológico e a menor rendimento acadêmico aos 18 anos.

Segundo alguns autores (Bhutta, Cleves, Casey, Cradock, \& Anand, 2002; Salt \& Redshaw, 2006), as dificuldades de aprendizagem e os problemas comportamentais, em crianças nascidas prematuras, tornam-se mais evidentes no início da idade escolar. Muitas vezes, de acordo com Amorim (2006), as crianças nascidas prematuras podem apresentar discretas alterações no primeiro ano de vida, como hipotonia axial e lentidão nas etapas motoras, porém, a partir de 18 a 24 meses, os sinais neurológicos podem não ser mais percebidos (alterações neurológicas transitórias). Portanto, para realizar intervenções efetivas, são necessários exames específicos e sensíveis que possibilitem a identificação de déficits cognitivos e motores leves, pois, mesmo na ausência de sinais perceptíveis ao exame neurológico tradicional, as crianças nascidas prematuras tendem a apresentar menores escores nos testes de inteligência e maior presença de TDAH e de distúrbios de comportamento (Johnson, 2007; Linhares, Chimello, Bordin, Carvalho, \& Martinez, 2005).

Espírito Santo, Portuguez e Nunes (2009), realizaram um estudo transversal brasileiro com 80 prematuros nascidos com IG $<37$ semanas e peso $<2.500 \mathrm{~g}$, onde utilizaram a Escala de Conners para Pais Revisada (Conners, 2001), com o objetivo de avaliar o comportamento dessas crianças aos 4-5 anos de idade. Nesse estudo, apesar das crianças terem nascido, predominantemente, com maior IG (61\% entre 34 e 36 semanas) e peso (69\% entre $1.500 \mathrm{~g}$ e $2.500 \mathrm{~g})$, foi observado alta taxa de problemas comportamentais.

Recém-nascidos com maior IG (de 34 a 36 semanas) também têm riscos de apresentar atraso no desenvolvimento, deficiência intelectual, encefalopatias e insucesso escolar. Petrini et al. (2009) conduziram um estudo populacional retrospectivo com 141.321 crianças nascidas com IG $>30$ semanas, sendo a maior parte da amostra $(92,7 \%)$ composta por crianças nascidas a termo. O objetivo da pesquisa era avaliar a associação entre prematuridade e incidência de sequelas neurológicas, em especial paralisia cerebral, atraso no desenvolvimento mental e presença de convulsão. Para a constatação de tais diagnósticos, os autores cruzaram os critérios da International Classification of Diseases, Ninth Revision, Clinical Modification (ICD-9 CM) com os dados de internação e ambulatorial de todas as crianças da amostra, que faziam parte do Kaiser Permanente Medical Care Program /EUA. Os autores constataram que a diminuição da IG foi associada com o aumento da incidência de paralisia cerebral e atraso no desenvolvimento mental, mesmo para aquelas crianças nascidas com prematuridade moderada, entre 34 e 36 semanas de gestação. Em um outro estudo (Chyi, Lee, Hintz, Gould, \& Sutcliffe, 2008), a aprendizagem de três grupos de crianças norte-americanas pré-escolares foi comparada, sendo o primeiro gru- 
po constituído por 203 crianças nascidas com IG entre 32 e 33 semanas; o segundo, formado por 767 prematuros nascidos entre 34 e 36 semanas de IG; e um outro que incluiu 13.671 crianças nascidas a termo. Todas faziam parte de um programa de seguimento e não apresentaram complicações neonatais significativas. $\mathrm{O}$ grupo nascido com menor IG teve pior resultado que os prematuros com IG entre 34 e 36 semanas, porém, os dois grupos de prematuros apresentaram mais dificuldade de aprendizagem e, por consequência, mais necessidade de educação especial, que os nascidos a termo. Os autores ressaltam, portanto, a importância de se acompanhar longitudinalmente as crianças nascidas prematuras, independentemente do fato de serem consideradas de menor risco para problemas do desenvolvimento.

A maioria das publicações não informa se há ou não adequação do peso ao nascer à IG. Sabe-se, entretanto, que os desvios de desenvolvimento nas crianças que nascem com muito baixo peso e/ou pequeno para a idade gestacional (PIG) têm repercussão, a longo prazo, e que as consequências podem se estender até a adolescência (Allin et al., 2006). No entanto, é importante destacar que prematuros nascidos AIG, embora possam apresentar melhor evolução clínica, também estão sujeitos às consequências mais tardias da prematuridade, evidenciadas no período escolar (Bayless \& Stevenson, 2007).

O presente estudo teve como objetivo comparar, aos sete anos de idade, o desempenho cognitivo e comportamental de crianças nascidas com até 33 semanas de gestação, peso inferior a $1.500 \mathrm{~g}$, porém AIG, e que apresentaram exame neurológico tradicional normal, com o de crianças da mesma idade, nascidas a termo e também AIG. Para as crianças nascidas prematuras, foi analisada a relação dos dados de um exame neurológico evolutivo (ENE) com os resultados do teste de inteligência e do questionário respondido pelos pais.

\section{Método}

Esta pesquisa foi realizada de abril de 2007 a junho de 2008, com delineamento transversal de comparação, envolvendo crianças nascidas prematuras no Hospital das Clínicas da Universidade Federal de Minas Gerais (HC/ UFMG) e acompanhadas no Ambulatório da Criança de Risco (ACRIAR). No ACRIAR, crianças nascidas com peso de até $1.500 \mathrm{~g}$ e/ou IG menor ou igual a 34 semanas são atendidas por uma equipe interdisciplinar, desde a alta neonatal até a idade de sete anos. Os dados dessas crianças foram comparados aos de outras nascidas a termo. O detalhamento dos critérios específicos de cada um dos grupos estão descritos adiante, em Participantes.

A avaliação neuropsicológica completa foi efetuada em três sessões de uma hora, para cada participante, de forma individual. O presente trabalho é uma análise de parte dos testes utilizados na avaliação neuropsicológica. $\mathrm{O}$ autor AFC foi o responsável pela avaliação neuropsicológica dos dois grupos com a contribuição da autora
JAN. O autor LFMD supervisionou a correção e a interpretação dos resultados dos dois grupos, sendo também o responsável pelas análises estatísticas. As crianças nascidas prematuras também foram submetidas, aos sete anos de idade, a exames neurológicos (tradicional e evolutivo), realizados pela autora RHCA, neuropediatra do ACRIAR. Os dois exames neurológicos foram feitos, preferencialmente, no mesmo dia. Esse estudo foi aprovado pelo Comitê de Ética em Pesquisa da UFMG.

\section{Participantes}

O grupo de prematuros foi composto por crianças nascidas no período de julho de 1999 a agosto de 2000, no HC/UFMG, e que permaneceram no ACRIAR até os sete anos. Essas crianças foram selecionadas à medida que faziam o exame neurológico tradicional no ACRIAR, aos sete anos, e atendiam aos critérios de inclusão: peso ao nascer inferior a $1.500 \mathrm{~g}$ e AIG; IG menor que 34 semanas; exame neurológico tradicional e perímetro cefálico (PC) normais, aos sete anos de idade, e ter quociente de inteligência (QI) entre 70 e 130, que corresponde à variação de até dois desvios-padrão em relação ao escore médio de 100 pontos. Esse grupo constou de 20 crianças com 7 anos a 7 anos e 10 meses de idade, das quais 13 (65\%) do sexo feminino e 7 (35\%) do sexo masculino, nascidas entre 27 e 33 semanas de gestação (média: 30 $\pm 1,8$ ) com peso de $770 \mathrm{~g}$ a $1.475 \mathrm{~g}$ (média: $1.178,50 \mathrm{~g} \pm$ $212,90 \mathrm{~g}$ ) e APGAR no quinto minuto de 3 a 10 (APGAR 3 , um caso; APGAR 10, um caso). A idade materna ficou entre 20 e 37 anos (média: $27 \pm 5$ anos), e a escolaridade da mãe variou de 4 a 11 anos de educação formal (média: $8 \pm 3$ anos). Quanto à presença de hemorragia periintraventricular (HPIV), enquanto 11 prematuros apresentaram ultrassom transfontanelar normal, três tiveram HPIV grau I da classificação de Papile (Papile, Burstein, Burstein, \& Koffler, 1978) e, em seis, o exame não foi realizado. As crianças deste grupo foram avaliadas no serviço de neuropsicologia do ACRIAR.

O grupo controle foi constituído de acordo com a necessidade de emparelhamento por idade, sexo e escolaridade com as crianças do ACRIAR. Os participantes desse grupo tinham entre 7 anos e 7 anos e 9 meses, nasceram a termo, com peso entre $2.550 \mathrm{~g}$ e $3.650 \mathrm{~g}$ (média: $3.097 \mathrm{~g} \pm 304 \mathrm{~g}$ ) e sem intercorrências clínicas ou cirúrgicas neonatais. Eles não apresentavam desvios de desenvolvimento, mencionados pelos pais e/ou professores. Todos os participantes desse grupo eram de uma única escola pública e foram avaliados na própria instituição. Permaneceram no estudo após constatação de QI na faixa de normalidade e de nível socioeconômico semelhante ao do grupo de prematuros, conforme o Critério de Classificação Econômica Brasil (Associação Brasileira de Empresas de Pesquisa [ABEP], 2008). Não foram obtidas informações sobre o APGAR no quinto minuto e nem o número preciso de semanas da gestação a termo. A idade materna variou de 17 a 39 anos (média: $26 \pm 7$ ) e o grau de instrução foi, em média, de $11 \pm 2,6$ anos. Como 
para essas crianças os exames neurológicos não foram realizados, adotaram-se como critérios de normalidade a ausência de intercorrências perinatais, desenvolvimento típico, comportamento e desempenho escolar considerado adequado pelas professoras e pelos pais.

Em ambos os grupos, as crianças eram AIG, frequentavam a primeira série de uma escola pública em Belo Horizonte/MG e não estavam em uso de medicamentos que atuam no sistema nervoso central (SNC). Crianças portadoras de má-formação cerebral ou ortopédica, microcefalia e/ou paralisia cerebral, déficit sensorial e/ ou cognitivo não foram incluídas na pesquisa.

\section{Instrumentos}

Avaliação Neurológica. Os exames neurológicos tradicional e evolutivo foram realizados, aos sete anos, pela neuropediatra do ACRIAR. A curva de perímetro cefálico utilizada foi a do National Center for Health Statistics NCHS (Hamill, Drizd, Johnson, Reed, \& Roche, 1977). O ENE - Lefèvre (Bacchiega, 1979; Lefèvre, 1976) é um método de investigação neurológica que consiste de provas simples, a maioria fundamentada na semiologia neurológica do adulto, porém com adaptações para se adequarem ao processo maturativo do SNC da criança.

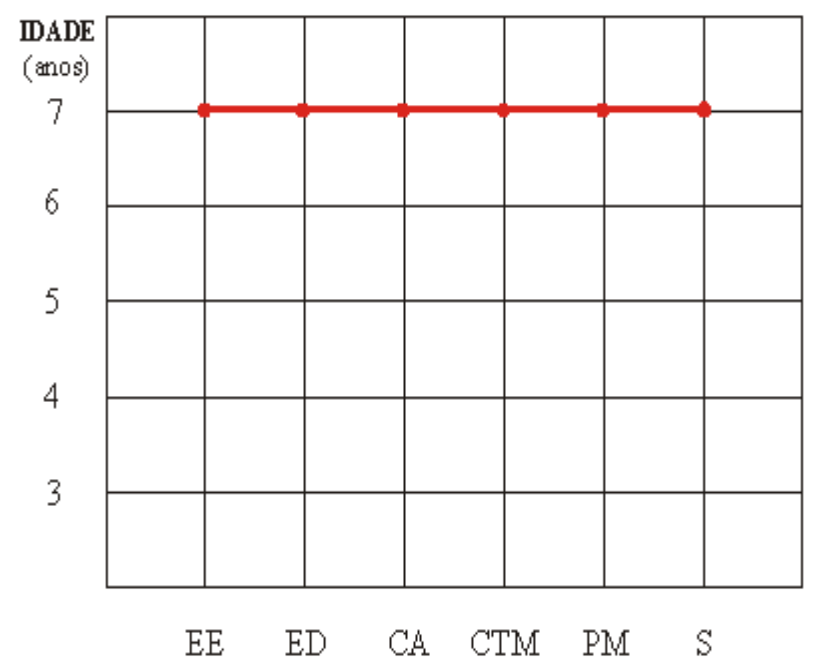

A
Existem diferentes provas para a faixa etária de três a sete anos, algumas realizadas com tempo determinado, que varia de 10 a 40 segundos. Essas provas avaliam equilíbrio estático (EE), equilíbrio dinâmico (ED), coordenação apendicular (CA), coordenação tronco e membros (CTM), persistência motora (PM), atividade sensitiva e sensorial (S). A lateralidade é também investigada, por meio de testes para a preferência ocular, manual e uso do pé. Cada prova é demonstrada à criança que, após compreendê-la, poderá fazer duas tentativas de realizála. Se ela não consegue fazer o item próprio de sua idade, propõe-se o da idade imediatamente inferior, até que a criança seja capaz de realizar a prova equivalente ao nível de maturidade das vias do SNC relacionadas com o que lhe é proposto. Quando a criança coopera e é participativa, a consulta dura, em média, de 30 a 40 minutos. No presente trabalho, a lateralidade não foi analisada. Os resultados obtidos constituem um gráfico denominado Perfil Neurológico de Desenvolvimento. Na Figura 1, estão representados dois exemplos do referido gráfico, que ilustram o desempenho de duas crianças da amostra, nascidas prematuras, sendo que uma delas (A) conseguiu fazer todas as provas propostas para sete anos de idade, $\mathrm{e}$ a outra (B), teve o desempenho alterado.

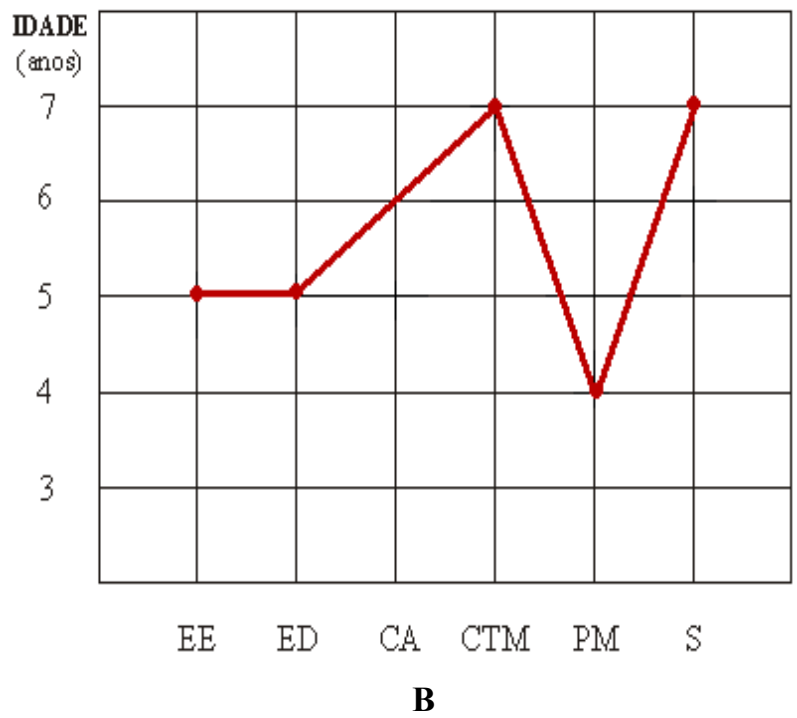

Figura 1. Perfil neurológico de desenvolvimento elaborado a partir da idade de execução das diferentes provas do ENE.

Nota. Em A, desempenho adequado para sete anos de idade; em B, desempenho alterado, pois a criança de sete anos de idade conseguiu executar adequadamente apenas as provas de Coordenação Tronco-membros (CTM) e de Atividade Sensitiva e Sensorial $(\mathrm{S})$.

Avaliação Neuropsicológica. Os pais das crianças de ambos os grupos preencheram, com a ajuda pesquisador principal, o Questionário de Hiperatividade para Pais de Conners (Barbosa, Dias, \& Gaião, 1997), que permite investigar problemas comportamentais em crianças. Esse instrumento é composto por 48 itens que identificam sintomas de desatenção, impulsividade e hiperatividade, sendo que cada afirmativa é pontuada por 0 (de modo algum); 1 (um pouco); 2 (bastante) e 3 (muito), de acordo com a intensidade que determinado sintoma é percebido na criança.

Todas as crianças do estudo foram submetidas à Escala Wechsler de Inteligência para Crianças III WISC-III (Figueiredo, 2002). Este instrumento permite a avaliação da inteligência total, inteligência verbal, inteligência de execução, velocidade de processamento, organização perceptiva, compreensão verbal e resistência à distração. 
Análise Estatística. Os resultados obtidos em cada instrumento de avaliação foram processados em análise descritiva das respectivas médias e do desvios-padrão. Em seguida, foi realizada a comparação entre os resultados da WISC-III e do Questionário de Conners das crianças nascidas prematuras e a termo. Para isso, a análise comparativa entre os dois grupos foi efetuada pelo teste não paramétrico Mann-Whitney. A escolha desse método decorreu do pequeno tamanho da amostra e da distribuição não normal dos resultados na maioria dos instrumentos, como indicado pelo teste Kolmogorov-Smirnov (Conover, 1980).

Além disso, foi realizada a correlação de Spearman (1907) entre as variáveis ENE e Inteligência; ENE e Questionário de Conners do grupo de prematuros. No entanto, como seis crianças do grupo de prematuros, além de não realizarem a prova de Persistência Motora de sete anos, se recusaram a fazer a prova da idade inferior, elas foram excluídas da análise estatística, somente desta prova. Para a análise das seis provas do ENE foram atribuídos pontos relativos à idade na qual a criança conseguiu realizar cada prova. Portanto, a criança que realizou todas as provas próprias para a idade, alcançou uma pontuação máxima de 42 pontos. Optou-se por essa associação, pois a idade com a qual a criança executa determinada prova equivale ao nível de maturidade das vias do SNC relacionadas com o que lhe é proposto naquela prova.

Tabela 1
Os dados obtidos foram submetidos a tratamento por meio do pacote estatístico Statistic Package Social for Science (SPSS, versão 15.0). O valor do nível de significância adotado é o inferior a 0,05 .

\section{Resultados}

\section{Amostra}

Em relação ao emparelhamento pelo nível socioeconômico, para se verificar a semelhança dos grupos, foi feita comparação dos pontos obtidos no Critério de Classificação Econômica Brasil (ABEP, 2008). Todas as crianças ficaram situadas entre as classes B2 e C2 e não foi constatada diferença com significância estatística entre os grupos $(p=0,154)$.

A escolaridade das mães e dos pais do grupo controle foi maior que a do grupo de prematuros, porém só houve diferença com significância estatística $(p=0,002)$ para a escolaridade materna ( $11 \pm 2,6$ anos, para as mães do grupo controle e $8 \pm 3$ anos, para as mães do grupo de prematuros).

\section{Exame Neurológico Evolutivo}

Apenas quatro crianças do grupo de prematuros (20\%) realizaram todas as provas do ENE para sete anos de idade. Nas demais, o número de provas alteradas, dentre as seis propostas, foi de uma a quatro por criança. Na Tabela 1 está apresentada a porcentagem de fracasso e sucesso nas provas do ENE.

Frequência de Realização das Provas do ENE

\begin{tabular}{|c|c|c|c|c|}
\hline \multirow[b]{3}{*}{ Provas ENE } & \multicolumn{4}{|c|}{ Grupo de prematuros $(n=20)$} \\
\hline & \multicolumn{2}{|c|}{ Fracasso } & \multicolumn{2}{|l|}{ Sucesso } \\
\hline & $n$ & $\%$ & $n$ & $\%$ \\
\hline Equilíbrio Estático & 06 & 30 & 14 & 70 \\
\hline Equilíbrio Dinâmico & 05 & 25 & 15 & 75 \\
\hline Coordenação Apendicular & 02 & 10 & 18 & 90 \\
\hline Persistência Motora & $05^{\mathrm{a}}$ & 36 & $09^{\mathrm{a}}$ & 64 \\
\hline Atividade Sensitiva e Sensorial & 01 & 5 & 19 & 95 \\
\hline Coordenação Tronco-membros & 05 & 25 & 15 & 75 \\
\hline
\end{tabular}

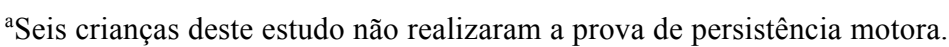

O PC das crianças nascidas prematuras, aos sete anos de idade, variou de $48 \mathrm{~cm}$ a $53 \mathrm{~cm}$, no sexo feminino, e de $50 \mathrm{~cm}$ a $53,9 \mathrm{~cm}$, no sexo masculino. Quatro crianças do sexo feminino e uma do sexo masculino tiveram a medida do PC entre os percentis p5 e p10, que são considerados normais, porém localizados no limite inferior dos valores de normalidade da curva do NCHS (Hamill et al., 1977). Dentre as cinco crianças do ACRIAR que tinham os menores PC's, entre os percentis p5 e p10, duas tiveram o pior desempenho no QI total, de 73 para um menino $(\mathrm{PC}=50 \mathrm{~cm})$ e de 71 , para uma menina $(\mathrm{PC}=48 \mathrm{~cm})$, valores estes pouco acima do limite inferior de normalidade. A criança do sexo masculino apresentava estatura entre os percentis p25 e p50 e peso entre p10 e p25, portanto, discordantes com o percentil do PC, que é menor. Já a criança do sexo feminino, que nasceu com o peso mais baixo deste grupo $(770 \mathrm{~g})$, tinha a estatura entre $\mathrm{p} 5$ e p10 e peso entre os percentis p3 e p5, proporcionais, portanto, ao percentil do PC, ficando difícil inferir se há discordância do crescimento cefálico. Em relação ao ENE dessas duas crianças, o perfil neurológico de desenvolvimento foi discrepante para a idade, em particular para a 
prova de persistência motora, com nível de quatro e cinco anos, respectivamente, para o menino e para a menina.

\section{Avaliação Neuropsicológica}

As médias e os desvios-padrão dos resultados da avaliação da inteligência e do comportamento, nos dois grupos, são mostrados na Tabela 2 .

Quanto à inteligência, apesar de todas as crianças do estudo apresentarem QI dentro do padrão de normalidade, o desempenho do grupo de crianças nascidas prematuras foi inferior ao do grupo controle, no QI Total, QI Verbal e QI Execução. Entretanto, não foram encontradas diferenças significativas entre o QI Verbal e QI Execução. Ainda em relação aos resultados da WISC-III, foram verificadas diferenças significativas na compreensão verbal e na velocidade de processamento, com pior performance para as crianças nascidas prematuras. Como já mencionado, a média de anos de escolaridade das mães das crianças nascidas a termo foi superior, o que pode ter contribuído para reforçar as diferenças encontradas entre os grupos, no que se refere ao desempenho cognitivo.

Aos sete anos, 13 crianças (65\%) do grupo de prematuros apresentavam queixas relatadas pelos pais, que incluíam déficit de atenção, hiperatividade e dificuldades escolares (seis casos); déficit de atenção e dificuldades escolares (dois casos); distúrbios emocionais e dificuldades escolares (um caso); comportamento desafiador (um caso); dificuldade em leitura e escrita (um caso); dificuldade em leitura (um caso); e dificuldade de interação (um caso). Apesar disso, não foram observadas diferenças com significância estatística em relação às médias obtidas pelo Questionário de Conners, conforme pode ser verificado na Tabela 2 .

\section{Correlações entre ENE, Avaliação da Inteligência} e do Comportamento

As provas do ENE, aplicado apenas nos prematuros, foram correlacionadas com a WISC-III e com o Questionário de Conners (Tabela 3).

Tabela 2

Avaliação Cognitivo-comportamental de Crianças Nascidas Prematuras e a Termo

\begin{tabular}{lrrrrr}
\hline \multirow{2}{*}{ Instrumentos } & \multicolumn{2}{c}{ Prematuros $(n=20)$} & Controle $(n=20)$ & Mann-Whitney \\
\cline { 2 - 4 } & $M$ & $D P$ & & \\
\hline WISC-III & & & & & \\
QI Total & 96,45 & 12,20 & 108,40 & 8,66 & 0,001 \\
QI Execução & 94,10 & 10,45 & 100,45 & 8,70 & 0,028 \\
QI Verbal & 99,80 & 12,39 & 114,55 & 9,29 & 0,000 \\
Compreensão Verbal & 99,25 & 11,00 & 114,15 & 10,83 & 0,000 \\
Organização Perceptiva & 91,25 & 9,93 & 95,90 & 9,20 & 0,121 \\
Resistência à Distração & 99,45 & 15,79 & 107,05 & 7,02 & 0,055 \\
Velocidade de Processamento & 104,15 & 8,60 & 110,75 & 7,50 & 0,010 \\
Questionário de Conners & 0,80 & 0,50 & 0,77 & 0,45 & 0,752 \\
\hline
\end{tabular}

Tabela 3

Correlação entre as Provas do ENE e a Avaliação Cognitivo-comportamental

\begin{tabular}{|c|c|c|c|c|c|c|}
\hline \multirow{2}{*}{ Instrumentos } & \multicolumn{6}{|c|}{ Provas do ENE $(n=20)$} \\
\hline & $\mathrm{EE}$ & ED & CA & PM & $\mathrm{S}$ & CTM \\
\hline \multicolumn{7}{|l|}{ WISC-III } \\
\hline QI Verbal & 0,228 & 0,403 & $0,462^{*}$ & 0,442 & 0,240 & 0,010 \\
\hline QI Execução & 0,042 & 0,331 & 0,444 & 0,187 & 0,140 & 0,060 \\
\hline QI Total & 0,070 & 0,375 & $0,461^{*}$ & 0,270 & 0,179 & 0,040 \\
\hline Compreensão Verbal & 0,236 & 0,429 & $0,475^{*}$ & 0,464 & 0,259 & 0,000 \\
\hline Organização Perceptiva & 0,109 & 0,340 & $0,458^{*}$ & 0,139 & 0,219 & 0,010 \\
\hline Resistência à Distração & 0,104 & 0,367 & 0,383 & 0,265 & 0,060 & 0,040 \\
\hline Velocidade de Processamento & $-0,248$ & 0,074 & 0,233 & 0,009 & 0,060 & 0,081 \\
\hline Questionário de Conners & $-0,112$ & $-0,051$ & $-0,368$ & $-0,289$ & $-0,060$ & 0,251 \\
\hline
\end{tabular}

Nota. Seis crianças não realizaram a prova de persistência motora. EE: equilíbrio estático; ED: equilíbrio dinâmico; CA: coordenação apendicular; PM: persistência motora; S: atividade sensitiva e sensorial; CTM: coordenação tronco-membros.

${ }^{*}$ Correlação de Spearman significativa $(p<0,05)$. 
Somente uma das provas do ENE, a de coordenação apendicular, apresentou correlação com o QI Verbal; o QI Total; a compreensão verbal e a organização perceptiva.

Houve significância estatística na correlação entre IG e QI Verbal ( $p=0,001)$, QI Total $(p=0,011)$, compreensão verbal $(p=0,001)$ e velocidade de processamento ( $p$ $=0,044)$. O baixo peso ao nascer teve correlação com o QI Verbal $(p<0,001)$, QI Execução $(p=0,039)$, QI Total $(p=0,001)$, compreensão verbal $(p<0,001)$ e velocidade de processamento $(p=0,002)$. Não se identificaram correlações significativas entre as demais variáveis perinatais com os resultados da avaliação neuropsicológica e com as provas do ENE.

\section{Discussão}

Apesar do conhecimento de que quanto menor o peso ao nascer e a IG, mais numerosas são as intercorrências neonatais e maiores os riscos de sequelas neurológicas e de desenvolvimento, nem sempre há relação direta entre os antecedentes perinatais e o desenvolvimento da criança. Por isso, é indispensável conhecer não só os fatores de risco biológico, como também os diagnósticos neurológico e psicológico, e a influência do nível socioeconômico e cultural na evolução e no comportamento de prematuros extremos.

No setor de saúde pública, da cidade de Belo Horizonte, são poucas as possibilidades de acompanhamento longitudinal de crianças que, nascidas prematuras, atingem a idade escolar com dificuldades geralmente não detectadas anteriormente. O ACRIAR, fundado em 1988, é um dos raros serviços brasileiros que estende o seguimento interdisciplinar até os sete anos de idade. Essas crianças são, na maioria, oriundas de uma população de baixa renda, e os pais têm pouca escolaridade. Ao longo do seguimento, essas crianças são submetidas a exames neurológicos em idades-chave até os sete anos. Embora com pequeno tamanho amostral, a importância desta pesquisa é ter investigado o desenvolvimento de crianças nascidas prematuras, com muito baixo peso, porém AIG e que, aos sete anos de idade, apresentavam exame neurológico, PC e inteligência normais. Além disso, estudos como o nosso, com crianças nascidas prematuras e AIG são pouco comuns, o que é preocupante, pois os riscos nessa população podem ser subestimados (Bayless \& Stevenson, 2007). Apesar dessas características favoráveis, de acordo com os resultados deste trabalho, embora na avaliação da inteligência, as crianças prematuras tenham conseguido pontuação dentro da média, as nascidas a termo obtiveram melhor desempenho no QI Total, QI Verbal e QI Execução, na compreensão verbal e na velocidade de processamento. As diferenças entre os grupos na escala de inteligência, mesmo quando estão dentro da normalidade, tem sido descritas de forma consistente na literatura (Anderson, Doyle, \& Victorian Infant Collaborative
Study Group, 2003; Bayless \& Stevenson, 2007; Esbjorn et al., 2006).

Um resultado interessante do presente estudo é a menor velocidade de processamento nas crianças prematuras. Johnson (2007), em artigo de revisão, constatou que o QI de crianças nascidas prematuras é inferior ao das nascidas a termo, mesmo quando aquelas não têm disfunções graves, e que também ocorrem desordens no raciocínio não-verbal e no processamento da informação.

Os resultados encontrados no ENE apontam a necessidade de análise mais minuciosa em crianças que foram prematuras, pois, mesmo apresentando exame neurológico tradicional normal, somente quatro indivíduos conseguiram executar todas as provas previstas para a idade. As dificuldades foram mais frequentes em persistência motora. A baixa ocorrência de dificuldade na prova de atividade sensitiva e sensorial, que compreende o conhecimento do lado direito e esquerdo do corpo, pode estar relacionada com a aprendizagem e o treinamento, pois são conceitos abordados no quotidiano escolar. Breslau et al. (2000) também detectaram disfunções neurológicas sutis em crianças com exame neurológico tradicional normal. No entanto, diferentemente do presente estudo, estes autores não mencionaram a IG da população analisada.

No presente estudo, embora os pais de seis crianças nascidas prematuras se queixassem, ao mesmo tempo, de déficit de atenção, hiperatividade e dificuldades escolares, em apenas dois casos o Questionário para Pais de Conners coincidiu com essas informações; um deles corresponde à criança do sexo masculino com o menor PC. Contrariando nossos resultados, Espírito Santo et al. (2009), identificaram comportamentos relacionados com TDA/H em 48\% da amostra de prematuros, aos 4-5 anos de idade, utilizando uma versão revisada da Escala de Conners para Pais. Contudo, cabe destacar que a referida pesquisa não contou com um grupo controle de crianças nascidas a termo para facilitar a interpretação dos resultados, além disso, a amostra foi bastante heterogênea em decorrência dos critérios de inclusão, somado ao fato de que 30 crianças não foram acompanhadas regularmente durante os primeiros anos de vida e 10 tinham alterações no exame neurológico na idade pré-escolar. Não se pode afirmar, no entanto, que essa discordância de resultados entre os estudos decorra de falha no Questionário de Conners, aliás, bastante usado no Brasil. Esse questionário pode complementar informações não obtidas com os pais ou durante o exame clínico da criança, porém, deve ser usado com critério.

Linhares et al. (2005) encontraram mais problemas comportamentais em crianças nascidas prematuras quando comparadas a um grupo de crianças nascidas a termo, entretanto, além de não mencionarem a adequação do peso de nascimento à IG, incluíram crianças com sequelas neurológicas graves e com déficit auditivo, o que pode ter influenciado os resultados. Ressalta-se, também, que o 
instrumento para avaliar o comportamento foi diferente do usado no presente estudo e os prematuros que constituíram nossa amostra foram acompanhados longitudinalmente no ACRIAR, o que pode ter interferido como um fator de proteção.

Uma das limitações do presente estudo foi a não realização do ENE no grupo controle. Porém, o ENE, padronizado e revisado, foi aplicado em crianças com desenvolvimento típico e nascidas a termo, como as do grupo controle deste trabalho. Entretanto, os resultados da nossa pesquisa são consistentes com aqueles previamente obtidos, por outros métodos, pelos pesquisadores do próprio ACRIAR. Diferenças significativas foram descritas nas habilidades perceptivas e motoras entre crianças nascidas prematuras e a termo na idade pré-escolar e escolar, com maior frequência de dificuldades nas nascidas prematuras (Magalhães, Catarina, Barbosa, Mancini, \& Paixão, 2003). Apesar do nível socioeconômico dos dois grupos se situarem entre classes semelhantes, nota-se que as mães das crianças do grupo controle tiveram média de escolaridade superior, o que pode ter influenciado no melhor desempenho cognitivo dessas crianças (Roberts, Bellinger, \& Mccormick, 2007).

Embora o ENE possa verificar problemas não evidenciáveis no exame neurológico tradicional, em especial, de coordenação motora e de equilíbrio, esse tipo de avaliação não tem como objetivo direto avaliar domínios cognitivos específicos. Ainda assim, observamos correlação positiva e com significância estatística entre as provas de coordenação apendicular e as medidas de inteligência. Como os sistemas fronto-estriatais estão relacionados à motricidade e às funções executivas (Diamond, 2000) e à inteligência (Shaw et al., 2006), estes resultados podem indicar a existência de substrato neurobiológico semelhante nas crianças do presente estudo, que tiveram desempenho deficitário.

Os dados da presente pesquisa alertam para a necessidade de avaliações mais específicas em crianças nascidas prematuras, ainda que sejam consideradas de menor risco para distúrbios ou aparentemente normais nos primeiros anos de vida, a fim de se investigarem possíveis dificuldades que venham a provocar prejuízos na vida diária, especialmente no contexto escolar. Os resultados dessas análises possibilitam a estruturação de intervenções comportamentais, psicoeducacionais e farmacológicas mais adaptadas ao contexto de vida da criança e que podem prevenir e/ou atenuar distúrbios de comportamento e de aprendizagem, na idade escolar e na adolescência. Conforme observado no ENE, mesmo com um perfil neurológico de desenvolvimento discrepante da idade cronológica, este exame deve ser complementado pela avaliação neuropsicológica, a qual, por meio de seus diferentes instrumentos, contribui para se estimarem as capacidades e as dificuldades da criança, adequando a abordagem terapêutica.

Estudos futuros, com amostras maiores e envolvendo medidas neuropsicológicas específicas, associadas a téc- nicas de neuroimagem, poderão elucidar a relação entre os diferentes sistemas neurais e os déficits cognitivos apresentados por indivíduos nascidos prematuros e com baixo peso. Os avanços tecnológicos para a preservação da vida e da função futura do SNC trarão novos desafios para os profissionais que atuam com crianças nascidas prematuras. É importante que déficits mais específicos do desenvolvimento, que podem não ser reconhecidos clinicamente ou não se manifestar nos primeiros anos de vida, em razão da própria imaturidade do SNC, sejam bem compreendidos por meio de avaliações sistematizadas. Para isso é necessária a implementação de políticas governamentais que estimulem o seguimento dessas crianças, até pelo menos os dez anos de idade e de modo abrangente, a vários serviços de saúde. Esses aspectos abrem, com certeza, perspectivas para novas pesquisas na população brasileira, em particular, com os recém-nascidos com peso inferior a $1.000 \mathrm{~g}$, ainda com sobrevivência restrita no país.

\section{Referências}

Achenbach, T. M. (1991a). Child Behavior Checklist for Ages 4-18. Burlington, VT: University of Vermont, Center for Children, Youth, \& Families.

Achenbach, T. M. (1991b). Teacher's Report Form. Burlington, VT: University of Vermont, Center for Children, Youth, \& Families.

Associação Brasileira de Empresas de Pesquisa. (2008). Critério de Classificação Econômica Brasil. Retrieved February 14, 2008, from http://www.abep.org

Allin, M., Rooney, M., Griffiths, T., Cuddy, M., Wyatt, J., Rifkin, L., et al. (2006). Neurological abnormalities in young adults born preterm. Journal Neurology, Neurosurgery and Psychiatry, 77(4), 495-499.

Amorim, R. H. C. (2006). Avaliação neurológica do lactente e acompanhamento do recém-nascido de risco. In L. F. Fonseca, J. M. F. Cunha, G. Pianetti, \& J. A. C. F. Val (Eds.), Manual de Neurologia Infantil (pp. 11-20). Rio de Janeiro, RJ: Guanabara Koogan.

Anderson, P. J., Doyle, L. W., \& Victorian Infant Collaborative Study Group. (2003). Neurobehavioral outcomes of schoolage children born extremely low birth weight or very preterm in the 1990s. JAMA: The Journal of the American Medical Association, 289(24), 3264-3272.

Aylward, G. P. (2005). Neurodevelopmental outcomes of infants born prematurely. Journal of Developmental and Behavioral Pediatrics, 26(6), 427-440.

Bacchiega, M. C. M. (1979). Exame neurológico evolutivo de crianças normais de 3 a 7 anos de idade: Contribuição para a avaliação de fidedignidade das provas. Dissertação de Mestrado não-publicada, Faculdade de Medicina, Universidade de São Paulo, SP.

Barbosa, G. A., Dias, M. R., \& Gaião, A. A. (1997). Validación factorial de los indices de hiperactividad del cuestionário de Conners en escolares de João Pessoa - Brasil. Revista de Neuropsiquiatria da Infância e Adolescência, 5(3), 118-125.

Bayless, S., \& Stevenson, J. (2007). Executive functions in school-age children born very prematurely. Early Human Development, 83(4), 247-254. 
Bhutta, A. T., Cleves, M. A., Casey, P. H., Cradock, M. M., \& Anand, K. J. (2002). Cognitive and behavioral outcomes of school-aged children who were born preterm: A metaanalysis. JAMA: The Journal of the American Medical Association, 288(6), 728-737.

Böhm, B., Smedler, A. C., \& Forssberg, H. (2004). Impulse control, working memory and other executive functions in preterm children when starting school. Acta Paediatrica, 93(10), 1363-1371.

Breslau, N., Chilcoat, H. D., Johnson, E. O., Andreski, P., \& Lucia, V. C. (2000). Neurologic soft signs and low birthweight: Their association and neuropsychiatric implications. Biological Psychiatry, 47(1), 71-79.

Briscoe, J., Gathercole, S. E., \& Marlow, N. (2001). Everyday memory and cognitive ability in children born very prematurely. Journal of Child Psychology and Psychiatry, 42(6), 749-754.

Buchanan, R. W., \& Heinrichs, D. W. (1989). The Neurological Evaluation Scale (NES): A structured instrument for the assessment of neurological signs in schizophrenia. Psychiatry Research, 27(3), 335-350.

Chyi, L. J., Lee, H. C., Hintz, S. R., Gould, J. B., \& Sutcliffe, T.L. (2008). School outcomes of late preterm infants: Special needs and challenges for infants born at 32 to 36 weeks gestation. Journal of Pediatrics, 153(1), 25-31.

Conners, C. K. (2001). Conners' rating scales-revised. North Tonawanda, NY: Multi-Health Systems.

Conover, W. J. (1980). Practical Nonparametric Statistics $\left(2^{\text {nd }}\right.$ ed.). New York: John Wiley and Sons.

Cooke, R. W. (2005). Perinatal and postnatal factors in very preterm infants and subsequent cognitive and motor abilities. Archives of Disease in Childhood. Fetal and Neonatal Edition, 90(1), F60-F63.

Cooke, R. W., \& Foulder-Hughes, L. (2003). Growth impairment in the very preterm and cognitive and motor performance at 7 years. Archives of Disease in Childhood, $88(6), 482-487$.

Diamond, A. (2000). Close interrelation of motor development and cognitive development and of the cerebellum and prefrontal cortex. Child Development, 71(1), 44-56.

Esbjorn, B. H., Hansen, B. M., Greisen, G., \& Mortensen, E. L. (2006). Intellectual development in a danish cohort of prematurely born preschool children: Specific or general difficulties? Journal of Developmental \& Behavioral Pediatrics, 27(6), 477-484.

Espírito Santo, J. L. do, Portuguez, M. W., \& Nunes, M. L. (2009). Status cognitivo-comportamental de prematuros de baixo peso ao nascimento em idade pré-escolar que vivem em país em desenvolvimento. Jornal de Pediatria, 85(1), $35-41$.

Figueiredo, V. L. M. (2002). Padronização brasileira das Escalas Wechsler de Inteligência para Crianças, Terceira Edição - WISC-III. In D. Wechsler, WISC-III: Escala de Inteligência para Crianças: Manual. São Paulo, SP: Casa do Psicólogo.

Fily, A., Truffert, P., Ego, A., Depoortere, M. H., Haquin, C., \& Pierrat, V. (2003). Neurological assessment at five years of age in infants born preterm. Acta Paediatrica, 92(12), 1433-1437.

Griffiths, T. D., Sigmundsson, T., Takei, N., Rowe, D., \& Murray, R. M. (1998). Neurological abnormalities in familial and sporadic schizophrenia. Brain, 121(2), 191-203.
Hamill, P. V., Drizd, T. A., Johnson, C. L., Reed, R. B., \& Roche, A. F. (1977). NCHS growth curves for children birth-18 years, United States. Vital Health Statistics, 11(165), i-iv, 1-74.

Hertzig, M. E. (1987). Neurologic evaluation schedule. In D. E. Tupper (Ed.), Soft Neurological Signs (pp. 355-368). Orlando, FL: Grune \& Stratton.

Isaacs, E. B., Edmonds, C. J., Lucas, A., \& Gadian, D. G. (2001). Calculation difficulties in children of very low birthweight: A neural correlate. Brain, 124(Pt. 9), 1701-1707.

Johnson, S. (2007). Cognitive and behavioural outcomes following very preterm birth. Seminars in Fetal and Neonatal Medicine, 12(5), 363-373.

Lefèvre, A. F. B. (1976). Exame neurológico evolutivo do préescolar normal (2. ed.). São Paulo, SP: Sarvier.

Linhares, M. B. M., Chimello, J. T., Bordin, M. B. M., Carvalho, A. E. V., \& Martinez, F. E. (2005). Desenvolvimento psicológico na fase escolar de crianças nascidas pré-termo em comparação com crianças nascidas a termo. Psicologia: Reflexão e Crítica, 18(1), 109-117.

Luu, T. M., Ment, L. R., Schneider, K. C., Katz, K. H., Allan, W. C., \& Vohr, B. R. (2009). Lasting effects of preterm birth and neonatal brain hemorrhage at 12 years of age. Pediatrics, 123(3), 1037-1044.

Magalhães, L. C., Catarina, P. W., Barbosa, V. M., Mancini, M. C., \& Paixão, M. L. (2003). Estudo comparativo sobre o desempenho perceptual e motor na idade escolar em crianças nascidas pré-termo e a termo. Arquivos de Neuropsiquiatria, 61(2A), 250-255.

McGrath, M., \& Sullivan, M. (2002). Birth weight, neonatal morbidities, and school age outcomes in full-term and preterm infants. Issues in Comprehensive Pediatric Nursing, 25(4), 231-254.

McGrath, M. M., Sullivan, M., Devin, J., Fontes-Murphy, M., Barcelos, S., DePalma, J. L., et al. (2005). Early precursors of low attention and hyperactivity in a preterm sample at age four. Issues in Comprehensive Pediatric Nursing, 28(1), 115.

Organização Mundial da Saúde. (1998). CID-10. Classificação estatística internacional de doenças e problemas relacionados à saúde (10. ed. rev.). São Paulo, SP: Editora da Universidade de São Paulo.

Papile, L. A., Burstein, J., Burstein, R., \& Koffler, H. (1978). Incidence and evolution of subependymal and intraventricular homorrhage: A study of infants with birth weights less than 1,500gm. The Journal of Pediatrics, 92(4), 529-534.

Petrini, J. R., Dias, T., McCormick, M. C., Massolo, M. L., Green, N. S., \& Escobar, G. J. (2009). Increased risk of adverse neurological development for late preterm infants. Journal of Pediatrics, 154(2), 169-176.

Roberts, G., Bellinger, D., \& Mccormick, M. C. (2007). A cumulative risk factor model for early identifation of academic difficulties in premature and low birth weighr infants. Maternal Child Health Journal, 11(2), 161-172.

Rourke, B. P. (1985). Neuropsychology of learning disabilities: Advances in subtype analysis. New York: Guilford Press.

Rourke, B. P., Fisk, J. L., \& Strang, J. D. (1986). NeuropsychologicalAssessment of Children: A treatmentoriented approach. New York: Guilford Press.

Saigal, S., Ouden, L. D., Wolke, D., Hoult, L., Paneth, N., Streiner, D. L., et al. (2003). School-age outcomes in children who were extremely low birth weight from four international population-based cohorts. Pediatrics, 112(4), 943-950. 
Campos, A. F., Malloy-Diniz, L. F., Nascimento, J. A. \& Amorim, R. H. C. (2011). Aspectos Neuropsicológico e Neurológico de Crianças Nascidas Prematuras e com Peso Inferior a 1.500 gramas.

Salt, A., \& Redshaw, M. (2006). Neurodevelopmental followup after preterm birth: Follow up after two years. Early Human Development, 82(3), 185-197.

Shaw, P., Greenstein, D., Lerch, J., Clasen, L., Lenroot, R., Gogtay, N., et al. (2006). Intellectual ability and cortical development in children and adolescents. Nature, 440(30), 676-679.

Shum, D., Neulinger, K., O’Callaghan, M., \& Mohay, H. (2008). Attentional problems in children born very preterm or with extremely low birth weight at 7-9 years. Archives of Clinical Neuropsychology, 23(1), 103-112.

Spearman, C. (1907). Demonstration of formulae for true measurement of correlation. The American Journal of Psychology, 18(2), 161-169.

Wechsler, D. (1974). Wechsler Intelligence Scale for ChildrenRevised Manual. New York: Psychological Corporation.

Wechsler, D. (1981). Wechsler Adult Intelligence Scale-Revised. New York: Psychological Corporation.

Wechsler, D. (1992). Wechsler Intelligence Scale for Children ( $3^{\text {rd }}$ ed.). Sidcup, UK: Psychological Corporation.

Wechsler, D. (1993). Wechsler Objective Reading Dimensions Test. Sidcup, UK: Psychological Corporation.

Wechsler, D. (1996). Wechsler Objective Numerical Dimensions. Sidcup, UK: Psychological Corporation.

Woodcock, R. W., \& Johnson, M. B. (1989). Woodcock-Johnson Tests of Achievement-WJ-R. Itasca, IL: The Riverside. 